\title{
ON THE EXISTENCE OF FIXED POINTS IN A TOTALLY ORDERED SET
}

\author{
F. T. METCALF AND T. H. PAYNE
}

\begin{abstract}
The Tarski fixed point theorem, concerning an isotone mapping of a partially ordered set into itself, is extended to mappings which are not necessarily isotone, but which must map a totally ordered set into itself. The key requirement is that the function be continuous (in a certain sense) whenever it is "decreasing'. Then $a \leqq f(a), f(b) \leqq b$, and $a \leqq b \Rightarrow$ the existence of a fixed point of $f$.
\end{abstract}

1. Introduction. Suppose that $S$ is a partially ordered set ("poset") which is conditionally complete (every bounded subset has a least upper bound and a greatest lower bound). A well-known fixed point theorem, due to Tarski (see, e.g., Garrett Birkhoff [1, p. 115]), asserts that, if $f$ is an isotone map of $S$ into $S$ (i.e., it preserves the partial ordering in $S$ ), then $a \leqq b, a \leqq f(a), b \geqq f(b) \Rightarrow \exists$ a fixed point of $f$ in $S$.

The fixed point question, when $f$ is not isotone, but such that it reverses the ordering in $S$, has recently been considered by A. Abian [2]. Abian assumes that $S$ is totally ordered, dense, and conditionally complete (definitions of these concepts are reviewed in $\S 2$ ). Then $f$ has a fixed point in $S$ if $f$ satisfies a type of continuity condition, and there exist $a, b \in S$ such that $a \leqq b, a \leqq f(a)$, and $b \geqq f(b)$. This situation is typified by a function, defined over the real numbers, which is nonincreasing and continuous. The graph of such a function is "forced" to intersect the diagonal somewhere between $a$ and $b$.

Likewise, if one returns to the case of $f$ nondecreasing, the situation is typified by a nondecreasing function defined over the real numbers. Since such a function has only upward jumps for discontinuities, the graph is again "forced" to intersect the diagonal between $a$ and $b$.

Using the above observations as a guide, the present paper treats the general situation when the function is not restricted to be monotone. Since some form of continuity is required when $f$ is nonincreasing, the setting will be a set $S$ which is totally ordered, dense, and conditionally complete. As before, the situation is made intuitively transparent by

Received by the editors April 15, 1971.

AMS 1970 subject classifications. Primary 06A05, 47H10; Secondary 06A23, 47H05. Key words and phrases. Fixed point, totally ordered set, conditionally complete. 
considering a function over the real numbers. If the function is unrestricted when "increasing," continuous when "decreasing," and there exist $a, b \in S$ such that $a \leqq b, a \leqq f(a), b \geqq f(b)$, then the graph of $f$ is "forced" to intersect the diagonal between $a$ and $b$.

2. The existence theorem. At this point, for the sake of completeness, it will be recalled that a partially ordered set $S$ is

(i) totally ordered if $a, b \in S \Rightarrow a \leqq b$ or $b \leqq a$,

(ii) dense if $a, b \in S$ and $a<b \Rightarrow \exists c \in S$ with $a<c<b$,

(iii) conditionally complete if every bounded subset of $S$ has a l.u.b. and a g.l.b.

These properties provide the necessary structure on the underlying set $S$, in order to obtain a fixed point theorem when the function $f$ may be nonincreasing. Thus, it will be assumed throughout that $S$ has properties (i), (ii), and (iii).

As indicated above, it appears that a continuity condition, of some sort, is required when the function is decreasing. The condition adopted here is that the function be Darboux when decreasing (i.e., such that all intermediate values are attained when the function decreases), and "nonoscillatory" from either the right or left. This last concept is made precise in the following definition.

Definition. A function $f: S \rightarrow S$ is said to be nonoscillatory from the right if, for each $x \in S$,

$$
\bigcap_{u>x ; u \in S} f([x, u]) \text { has at most one point. }
$$

Likewise, $f$ is said to be nonoscillatory from the left if, for each $x \in S$,

$$
\bigcap_{u<x ; u \in S} f([u, x]) \text { has at most one point. }
$$

It may readily be verified that a monotone function is nonoscillatory from both the right and the left. Also, it should be noted that this concept reflects a rather general view of "nonoscillation." For example, the realvalued function given by

$$
\begin{aligned}
f(x) & =0, & & x \text { rational, } \\
& =1+x, & & x \text { irrational, }
\end{aligned}
$$

is nonoscillatory from both the right and left.

THEOREM. Suppose $f: S \rightarrow S$ satisfies

(1) $[f(y), f(x)] \subseteq f([x, y])$ whenever $x, y \in S, x \leqq y$, and $f(y) \leqq f(x)$,

(2) $f$ is nonoscillatory from either the right or left,

(3) $\exists a, b \in S$ such that $a \leqq b, a \leqq f(a), f(b) \leqq b$.

Then $f$ has a fixed point in the interval $[a, b]$. 
Proof. Suppose that $f$ is nonoscillatory from the right. Let

$$
A=\{x \mid a \leqq x \leqq b \text { and } w \leqq f(w), \forall w \in[a, x]\}
$$

and $c=\sup A$.

It will first be shown that $c \leqq f(c)$. Suppose, to the contrary, that $f(c)<c$; and let $w \in A \cap(f(c), c]$ (if this intersection were empty, then $f(c)$ would be an upper bound for $A$, contradicting $c=\sup A$ ). Then one has $f(c)<w \leqq$ $f(w)$; and hence,

$$
(f(c), w) \subseteq[f(c), f(w)] \subseteq f([w, c]),
$$

where (1) has been used to obtain the second containment. Thus, for $y \in(f(c), w)$, there exists $z \in[w, c) \subseteq A$ such that $y=f(z)$. This yields $f(z)=$ $y<w \leqq z$, or $f(z)<z$, contradicting $z \in A$.

Next it will be shown that $f(c) \leqq c$. Suppose, to the contrary, that $c<f(c)$, and let

$$
B=\{x \mid c \leqq x \leqq f(c) \text { and } f(x)<x\},
$$

so that $c=\inf B$. Then, for $x \in B, f(x)<x \leqq f(c)$, so that (1) yields

$$
[x, f(c)] \subseteq[f(x), f(c)] \subseteq f([c, x]), \quad \forall x \in B .
$$

For $x \in B$, the sets $[x, f(c)]$ increase as $x$ decreases, while the sets $f([c, x])$ decrease as $x$ decreases. Thus,

$$
(c, f(c)]=\bigcup_{x \in B}[x, f(c)] \subseteq \bigcap_{x \in B} f([c, x]) ;
$$

however, the intersection on the right has at most one element since $f$ is nonoscillatory from the right, which contradicts $c<f(c)$.

Finally, one has $c \leqq f(c)$ and $f(c) \leqq c$, so that the existence of a fixed point is established if $f$ is nonoscillatory from the right. When $f$ is nonoscillatory from the left, a similar argument yields the fixed point, this time starting with the set $A=\{x \mid a \leqq x \leqq b$ and $f(w) \leqq w, \forall w \in[x, b]\}$. Alternatively, the above result could be applied to the dual of $S$ in order to handle nonoscillation from the left.

RemarK 1. Simple examples, using functions defined on the real numbers, may be utilized to show that denseness, conditional completeness, and hypothesis (1) may not be individually dropped. For example, the function given by $f(x)=x^{2}+x-2$ maps rational numbers into rational numbers, yet has no rational fixed points; thus showing that conditional completeness may not be dropped. Of more interest though, is the question of dropping hypothesis (2) concerning nonoscillation. The following example shows that this hypothesis cannot be deleted.

Let $S$ be the closed real interval $[0,1]$. For $x \in[0,1]$, let $b_{0} \cdot b_{1} b_{2} b_{3} \cdots$ denote the binary expansion of $x$ (where the usual convention is followed 
i.e., the expansion is terminated whenever possible). Define $f$ at $x$ as follows:

$$
\begin{array}{ll}
1-\lim _{n \rightarrow \infty} \frac{1}{n} \sum_{i=1}^{n} b_{i} & \text { if the limit exists and } f(x) \neq x, \\
0 & \text { otherwise. }
\end{array}
$$

Then, in any open interval, $f$ assumes all values in $[0,1]$, so that the intermediate value hypothesis (1) is satisfied. However, it is clear that hypothesis (2) concerning nonoscillation is not satisfied from either the right or left. Also, by construction, $f$ has no fixed points in $[0,1]$.

REMARK 2. It is readily verified that the above theorem yields, as a special case, the result of Abian [2]. One need only check that if

$$
f(\sup A)=\inf f(A) \text { and } f(\inf B)=\sup f(B)
$$

for any subsets $A$ and $B$ of $S$, and $x \leqq y \Rightarrow f(y) \leqq f(x)$ whenever $x, y \in S$, then conditions (1) and (2) of the theorem are satisfied. Condition (1) is a consequence of the fact that the image of $[x, y]$, under the continuous function $f$, must be connected, i.e., an interval; but, this interval contains $f(x)$ and $f(y)$, and hence, the interval $[f(y), f(x)]$. Condition (2) follows from

$$
\begin{aligned}
\bigcap_{u>x} f([x, u]) & =\bigcap_{u>x}[f(u), f(x)]=\left[\sup _{u>x} f(u), f(x)\right] \\
& =[f(\inf u), f(x)]=[f(x), f(x)] .
\end{aligned}
$$

\section{BIBLIOGRAPHY}

1. Garrett Birkhoff, Lattice theory, 3rd ed., Amer Math. Soc. Colloq. Publ., vol. 25, Amer. Math. Soc., Providence, R.I., 1967. MR 37 \#2638.

2. Alexander Abian, $A$ fixed point theorem for nonincreasing mappings, Boll. Un. Mat. Ital. (4) 2 (1969), 200-201. MR 39 \#5427.

Department of Mathematics, University of California, Riverside, California 92502 